\title{
Telomere Shortening in Alveolar Macrophages of Smokers and COPD Patients
}

\author{
Katsuyuki Tomita ${ }^{*}, 1$, Gaetano Caramori ${ }^{2}$, Kazuhiro Ito $^{3}$, Sam Lim $^{3}$, Hiroyuki Sano ${ }^{1}$, Yuji Tohda ${ }^{1}$, \\ Ian M. Adcock ${ }^{3}$ and Peter J. Barnes ${ }^{3}$ \\ ${ }^{I}$ Department of Respiratory Medicine and Allergology, Kinki University School of Medicine, Osaka, Japan \\ ${ }^{2}$ Centro di Ricerca su Asma e BPCO, Università di Ferrara, Ferrara, Italy \\ ${ }^{3}$ Airways Disease Section of Thoracic Medicine, National Heart and Lung Institute at Imperial College School of \\ Science, Technology and Medicine, London, UK
}

\begin{abstract}
Background: Tobacco smoking is reported to enhance telomere shortening in peripheral blood lymphocytes. However, telomere length of lung resident cells such as alveolar macrophages (AMs) has not been evaluated.

Aim: We evaluated effect of tobacco smoking on telomere length in AMs.

Methods: AMs were obtained from nine non-smokers (mean age 24.6 years, mean $\mathrm{FEV}_{1} / \mathrm{FVC} 93.8 \%$ ) and ten healthy smokers (mean age 25.2 years, $>10$ pack-years, mean $\mathrm{FEV}_{1} / \mathrm{FVC} 90.6 \%$ ). We also analyzed tissue macrophages from eight moderate to severe COPD patients (mean age 68.3 years, $>10$ pack-years, mean $\mathrm{FEV}_{1} / \mathrm{FVC} 54.2 \%$, mean $\%$ pred. $\mathrm{FEV}_{1} 48.8 \%$ ), age-matched nine healthy smokers (mean age 67.1 years, $>10$ pack- years, mean $\mathrm{FEV}_{1} / \mathrm{FVC} 83.7 \%$, mean $\%$ pred. $\mathrm{FEV}_{1} 82.4 \%$ ), and eight healthy non-smokers (mean age 71.2 years, mean $\mathrm{FEV}_{1} / \mathrm{FVC} 84.6 \%$, mean $\%$ pred. $\mathrm{FEV}_{1}$ $84.1 \%$ ). Centromere signals and telomere length were analysed using quantitative fluorescence in situ hybridization (QFISH) and confocal microscopy.

Results: Telomere length was reduced in bronchoalveolar lavage macrophages of younger smokers compared with that observed in healthy non-smoker's AMs, Importantly, there was no quantitative difference in centromere signals between smokers and non-smokers. A shorter telomere length was also seen in tissue macrophages from older smokers with or without COPD compared with non-smokers. However there was no difference in telomere length between healthy smokers and smokers with COPD.
\end{abstract}

Conclusion: Tobacco smoking enhances telomere shortening in AMs from patients with and without COPD.

Keywords: Alveolar macrophages, COPD, senescence, smoking, telomere length.

\section{INTRODUCTION}

Alveolar macrophages (AMs) play a fundamental role in lung defense [1]. The life span of AMs from non-smokers is around 80 days [2] and this increases to almost two years in smokers [3]. The number of AMs from smokers is also increased in the airway. We have previously demonstrated that the increased numbers of AMs in the lower airways of smokers may result from increased recruitment and proliferation as well as reduced cell death as apoptotic cell death [4].

Telomeres are present at the ends of chromosomes and consist of up to 10 to 20 kilo bases of hexametric nucleotide repeats (TTAGGG) $)_{n}$ [5]. The average length of telomere repeats in most normal human somatic cells decrease by 50 200 base pairs with each cell division, thus, the telomere has been hypothesized to serve as a "molecular clock" that tallies the number of cell divisions and limits further divisions at a predetermined point [6].

*Address correspondence to this author at the Department of Respiratory Medicine and Allergology, Kinki University School of Medicine, 377-2 Ohno-higashi, Osakasayama 589-8511, Japan; Tel: +72-366-0221; Fax:+72-367-7772; E-mail: tomo223@ko-arena.med.kindai.ac.jp
Oxidative stress is a contribution of stress-induced damage to cellular DNA through the role of damage to telomeric DNA and in vitro to telomere-driven cell senescence [7]. Progressive telomere shortening in vivo has been observed in regions susceptible to age-associated diseases such as atherosclerosis [8,9], and vascular dementia [10]. Telomere attrition in blood leukocytes has been shown to be accelerated by smoking $[11,12]$ and traffic pollution [13].

In lung diseases, patients with idiopathic interstitial pneumonias (IIPs) $[14,15]$ and chronic obstructive pulmonary disease (COPD) [16] have shortened telomeres in circulating leukocytes. Pulmonary emphysema, a major component of COPD, is pathologically characterized by destructive alterations in pulmonary architectures as a result of persistent inflammation and sustained oxidative stress as a result of long-term exposure to cigarette smoking [17]. In addition, Tsuji and colleagues have reported that epithelial cells from patients with emphysema showed telomere length shortness [18].

The aim of our study was to investigate if AMs from smokers with or without airway obstruction had a reduced telomere length. To test this, we measured telomere length in 
bronchoalveolar lavage macrophages and tissue macrophages from non-smokers and from smokers with and without COPD using quantitative fluorescence in situ hybridization methods (QFISH) and confocal microscopy.

\section{MATERIALS AND METHODOLOGY}

\section{Study Population}

Nineteen healthy subjects were recruited for the study of bronchoalveolar lavage (BAL). Nine subjects [mean $( \pm \mathrm{SD})$ age $24.6 \pm 2.2$ years] were never-smokers with normal lung function and ten age-matched subjects were current smokers with a cumulative smoking history of at least 10 pack-years (Table 1). Eight patients with COPD [mean $( \pm$ SD) age 68.3 \pm 5.4 years] diagnosed according to GOLD guidelines [19], nine age-matched current smokers without airway obstruction $\left(\mathrm{FEV}_{1}>30 \%\right.$ predicted) and eight age-matched nonsmokers with normal lung function were also recruited (Table 2). All patients underwent elective bronchoscopy for diagnostic purposes whilst normal subjects were volunteers. Smokers, with or without COPD, had a smoking history of $>20$ pack years.

Table 1. Clinical Data of Subjects in Bronchoalveolar Lavage Study

\begin{tabular}{|c|c|c|}
\hline & $\begin{array}{c}\text { Non-Smokers } \\
\quad(n=9)\end{array}$ & $\begin{array}{c}\text { Smokers } \\
(n=10)\end{array}$ \\
\hline Age, yr & $24.6 \pm 2.2$ & $25.2 \pm 3.1$ \\
\hline Sex, M:F & $5: 4$ & $6: 4$ \\
\hline Pack-years & 0 & $14.3 \pm 3.1$ \\
\hline \multicolumn{3}{|l|}{ Spirometry } \\
\hline post-bronchodilator $\mathrm{FEV}_{1}, \%$ pred. & $98.8 \pm 2.8$ & $88.4 \pm 6.1$ \\
\hline post-bronchodilator FVC, \%pred. & $105.3 \pm 2.4$ & $97.6 \pm 3.8$ \\
\hline
\end{tabular}

Definition of abbreviations: $\mathrm{F}=$ female, $\mathrm{M}=$ male, $\mathrm{FEV}_{1}=$ forced expiratory volume in one second, $\mathrm{FVC}=$ forced vital capacity, pred. $=$ predicted.

Data are shown as mean $\pm \mathrm{SD}$.

COPD patients receiving inhaled corticosteroids or oral theophylline and those with an acute exacerbation in the previous 6 weeks were excluded. In addition, exclusion criteria for smokers and nonsmokers included suspicion of infective or interstitial disease. The subjects were non-atopic (i.e., they had negative skin tests for common allergen extracts) and had no past history of asthma or allergic rhinitis. Carbon monoxide (CO) was measured by exhaled $\mathrm{CO}$ measurements (NIOX, Aerocrine AB, Stockholm, Sweden) [20]. All patients gave written informed consent before participating in the study, which was approved by the Royal Brompton Hospital Ethics Committee.

\section{Fiberoptic Bronchoscopy and Bronchoalveolar Lavage}

Fiberoptic bronchoscopy was performed according to established guidelines [21]. The bronchoscope was wedged in the right middle lobe and four successive aliquots each of $60-\mathrm{ml}$ aliquots of pre-warmed sterile $0.9 \% \mathrm{NaCl}$ solution were instilled. The recovered BAL fluid was pooled and filtered through one layer of gauze, and the cells were separated by centrifugation for $10 \mathrm{~min}$ at $4^{\circ} \mathrm{C}$. The total cell number was determined in a hemocytometer, and cytocentrifuge preparations were stained with MayGrünwald-Giemsa stain for differential cell counts.

\section{Lung Biopsy Collection}

Biopsy specimens were gently extracted from the forceps and processed for light microscopy as previously described [22]. Briefly, samples were fixed in 4\% formaldehyde for $4 \mathrm{~h}$ and embedded in paraffin. The best specimen was then oriented and $4 \mu \mathrm{m}$ serial sections were cut. Two sections at an interval of $100 \mu \mathrm{m}$ were then appropriately stained for telomeres.

\section{Quantitative Fluorescence In Situ Hybridization (QFISH)}

The gold standard measurement of telomere length remains the analysis of terminal restriction fragment (TRF) length by Southern blot analysis [23]. However, the TRF does not indicate the exact telomere repeat length. On the other hand, the fluorescence intensity detected by quantitative fluorescence in situ hybridization (QFISH) is directly proportional to the size of telomere repeats. Furthermore, the QFISH technique shows a high degree of sensitivity, since this technique is able to detect differences in individual telomere lengths below $1 \mathrm{~kb}$ [24]. In this study, telomere signals were detected using QFISH as described by the Manufacturer (Dako, Ely, UK). In brief, paraformaldehyde-fixed AMs on slides were denatured in $70 \%$ formamide at $80^{\circ} \mathrm{C}$ for $3 \mathrm{~min}$ with a telomere-specific PNA probe/Cy3. Sections were hybridized for $1 \mathrm{~h}$ at room temperature before the slides were washed twice in rinse solution and once in wash solution. After dehydration, the sections were stained with 4', 6-diamidino -2-phenylindole (DAPI). The slides were mounted with anti-fade solution (Vector Laboratories, Burlingame, CA).

\section{Hybridization Probe Access Control}

To rule out differences in probe penetration or target accessibility as potential sources of observed differences in fluorescent telomere signal intensities in fixed samples, we used a rhodamine-labeled probe targeted at the centromeric region of chromosome 8 (CEN8) (Micro System, Spånga, Sweden) [25]. The probe was denaturated with the slide at $80^{\circ} \mathrm{C}$ for $3 \mathrm{~min}$ and hybridized for 30 to $60 \mathrm{~min}$ at room temperature. After dehydration, nuclei were counterstained with anti-fade solution containing DAPI for identification. The slides were mounted with anti-fade solution.

\section{Telomere and Centromere Image Analysis}

Digitized images of the slides were obtained with a confocal laser scanning (Leica, Heidelberg, Germany) equipped with a 488/512-nm dual-band argon ion laser. For all slides, the instrument settings remained constant. $Z$ series through the cell images were captured with a step size of $0.75 \mu \mathrm{m}$ per image; $5 \mathrm{z}$ series images were taken per field. Although the thin optical slices analyzed by confocal microscopy minimized the influence of background autofluorescence, compensation for local background fluorescence was still required. Firstly, color digitized images of red channel were transformed into black-white image and then inverted. QFISH analyses of telomere and centromere were performed with NIH image software for the Macintosh (Scanlytics, Fairfax, VA). As signals from the telomere and centromere probes were expressed at high pixel intensity, we analyzed 
the intensity of red signals as the area under the standardized curve fitted to the signals, following adjustment for noise (Fig. 2). Within each nucleus in each subject, the watershed algorithm is used to identify spots from telomere or centromere signals. We analysed telomere or centromere lengths using a spot-finding method, which was demonstrated by O'Sullivan and colleagues [26]. The background red fluorescence was calculated as the average intensity of all non-spot pixels within each nucleus under consideration, and this value was subtracted from each pixel within a spot to yield background corrected red within-spot pixel intensities.

\section{Statistical Analysis}

The results were shown as the mean \pm standard deviation (SD). The statistical analyses were performed with Instat 3 (Graph Pad Software Inc., San Diego, CA). Comparison between specific groups was made by Mann-Whitney nonparametric test. Among-groups comparison was examined by Kruskal-Wallis nonparametric analysis, followed by Dunn's procedure for post-hoc multi-comparison analysis.

\section{RESULTS}

\section{Clinical Data}

The clinical characteristics of subjects in the BAL study data are shown in Table 1, whilst those in the bronchoscopy study are shown in Table 2. Subjects in the respective groups were matched by age. The values of exhaled $\mathrm{CO}$ were higher in smokers $(16 \pm 11 \mathrm{ppm})$ than non-smokers $(2 \pm 3 \mathrm{ppm})(p<$ $0.01)$. In addition, the total cell number in BAL was higher in smokers than non-smokers $(p<0.01)$ (Table 3 ). In the bronchial biopsy study, the three groups of subjects were similar with regard to age and there was no significant difference in the smoking history between COPD patients and healthy smokers. As expected from the selection criteria, smokers with COPD had a significantly lower post-bronchodilator $\mathrm{FEV}_{1}$ (percentage of predicted) and post-bronchodilator $\mathrm{FVC}$ ratio as compared to smokers $(p<0.01)$ and non-smokers $(p<0.01)$ (Table 2).

Table 2. Clinical Data of Patients in Lung Biopsy Study

\begin{tabular}{|l|c|c|c|}
\hline & $\begin{array}{c}\text { COPD } \\
\text { Patients } \\
(\mathbf{n}=\mathbf{8})\end{array}$ & $\begin{array}{c}\text { Healthy } \\
\text { Smokers } \\
(\boldsymbol{n}=\mathbf{9})\end{array}$ & $\begin{array}{c}\text { Non-Smokers } \\
(\boldsymbol{n}=\mathbf{8})\end{array}$ \\
\hline \hline Age, yr & $68.3 \pm 5.4$ & $67.1 \pm 6.1$ & $71.2 \pm 6.4$ \\
\hline Sex, M:F & $6: 2$ & $6: 3$ & $6: 2$ \\
\hline Pack-years & $46.6 \pm 31.4$ & $42.8 \pm 25.9$ & 0 \\
\hline Spirometry & \multicolumn{3}{|l|}{$84.1 \pm 5.2$} \\
\hline \multicolumn{1}{|l|}{$\begin{array}{l}\text { post-bronchodilator } \\
\text { FEV }\end{array}$, \%pred. } & $48.8 \pm 8.2^{*}$ & $82.4 \pm 4.3$ & $99.2 \pm 1.8$ \\
\hline $\begin{array}{l}\text { post-bronchodilator } \\
\text { FVC, \%pred. }\end{array}$ & $90.2 \pm 4.8^{*}$ & $98.3 \pm 2.1$ & \\
\hline
\end{tabular}

Telomere Length in Bronchoalveolar Lavage Macrophages from Smokers and Non-Smokers Detected with QFISH

To demonstrate smoking-related telomere length dynamics in alveolar macrophages, we introduced a novel method of measuring telomere length by QFISH using PNA labeled probe. QFISH showed a telomere signal in the nuclei of bronchoalveolar macrophages from non-smokers. In contrast, a telomeric signal was seen in the cytoplasm as well as the nuclei of bronchoalveolar macrophages obtained from smokers (Fig. 1). As AMs from smokers had a green autofluorescence in the cytoplasm the algorithm was required in order to minimize the influence of background autofluorescence. The intensity histogram demonstrated that the signals from the telomere probe were distinguishable from noise and background (Fig. 2). The intensity of signals in each nucleus was analyzed using the area under the curve. When the telomere length was assessed using spot-finding method, the signal was significantly less intense in bronchoalveolar macrophages from smokers than in those from non-smokers (Fig. 3, $p<0.05$ ). In contrast, there was no significant deference in centromere length in bronchoalveolar macrophages between two groups when assessed using the same method (Fig. 4).

Table 3. Total and Differential Count in BAL

\begin{tabular}{|c|c|c|}
\hline & Non-Smokers $(n=9)$ & Smokers $(n=10)$ \\
\hline \multicolumn{3}{|l|}{ Cell content of BALF } \\
\hline Total cell count, $\mathrm{X} 10^{6}$ & $19.4 \pm 1.4$ & $31.3 \pm 3.6^{*}$ \\
\hline Macrophages, $\%$ & $92.5 \pm 2.3$ & $87.4 \pm 3.5$ \\
\hline Lymphocytes, \% & $6.4 \pm 2.1$ & $8.2 \pm 2.4$ \\
\hline Neutrophils, \% & $0.6 \pm 1.2$ & $1.6 \pm 1.1$ \\
\hline
\end{tabular}

Definition of abbreviations: $\mathrm{BAL}=$ bronchoalveolar lavage.

Data are shown as mean \pm SD. ${ }^{*} P<0.01$.

\section{Telomere Length in Tissue Macrophages from COPD and Healthy Controls Detected with QFISH}

The in situ analysis of telomere lengths of tissue macrophages from lung biopsies by confocal microscopy is illustrated in Fig. (5). Lung tissue had an intrinsic greenfluorescence in all three groups due to the presence of collagen. Higher autofluorescence in the cytoplasm of tissue macrophages was also shown in biopsy sections from agematched smokers with or without COPD patients compared with non-smokers. Tissue macrophages were identified using doubled staining by the May-Grünward method. Quantification of telomere expression demonstrated that tissue macrophages from COPD patients, as well as agematched smokers without COPD, had decreased fluorescence signals compared with age-matched nonsmokers (Fig. 6, $p<0.05$ ). A comparison between younger and older healthy non-smokers (compare Fig. 3 and Fig. 6) shows a reduced telomeric length in the older patients.

\section{DISCUSSION}

This study demonstrated a 1.6-fold shorter telomere length in bronchoalveolar macrophages obtained from younger smokers when compared to that from non-smokers when measured by QFISH (Fig. 3). We also showed a significantly shorter telomere length in tissue macrophages in smokers with and without COPD patients compared with age-matched non-smokers (Fig. 6) and a reduction in teleomere length in the older healthy subjects compared to 
younger subjects. In contrast, we did not observe any difference in centromere length between any of the subject groups.

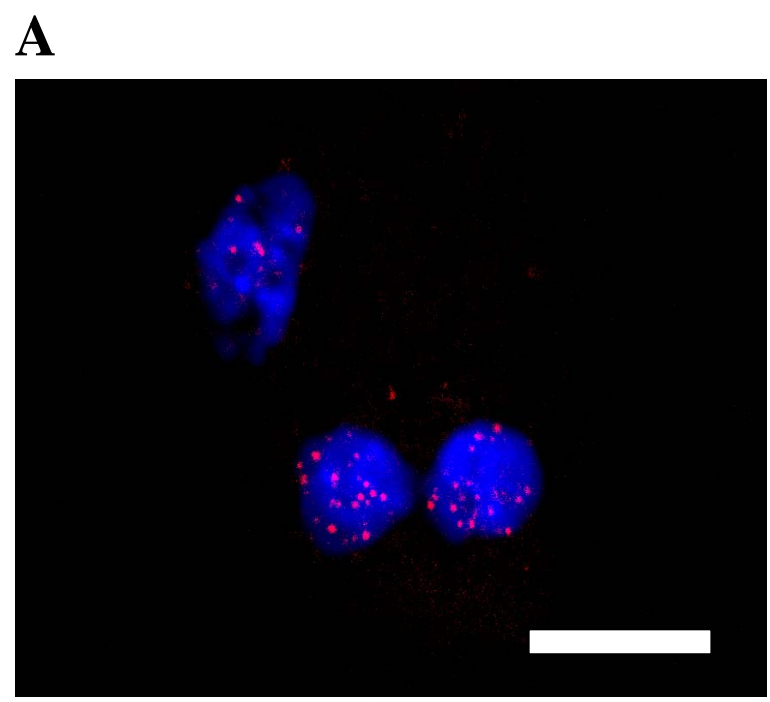

\section{B}

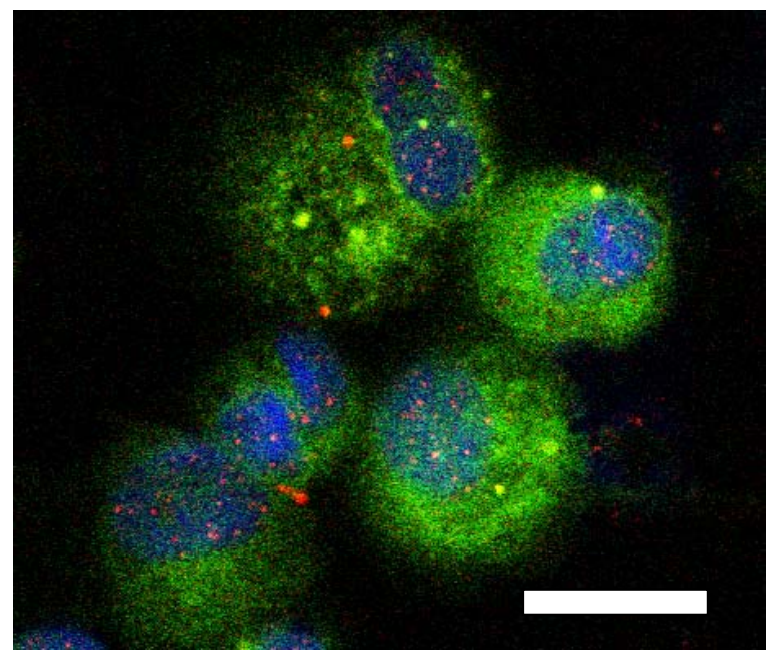

Fig. (1). Representative confocal microscope images of quantitative fluorescence in situ hybridization (QFISH) using telomere (red) probe counterstained with a fluorescent DNA dye, DAPI, in cytospins of alveolar macrophages (AMs) from non-smokers (A) and smokers (B). Red indicates telomere signals obtained with Cy3labeled $(\mathrm{CCCTAA})_{3}$ PNA, and blue indicates DAPI-stained chromosomal DNA. Scale bar $=20 \mu \mathrm{m}$ for each panel.

When we analyzed telomere length using the spot-finding method, the red channel could distinguish signals from telomere probe to noise from autofluorescence by pixel size. AMs from smokers have a bright autofluorescence mainly in the green channel and this may potentially interfere with the telomere signal. However, we demonstrated using the same technique that the centromere length in AM macrophages from smokers was similar to that from non-smokers (Fig. 4). This suggests, therefore, that the differences in telomere
A

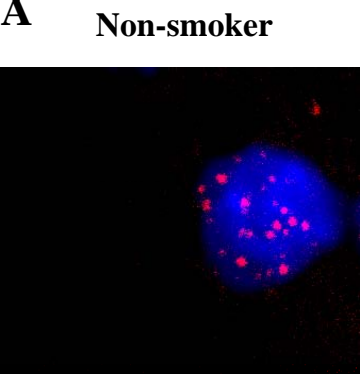

B
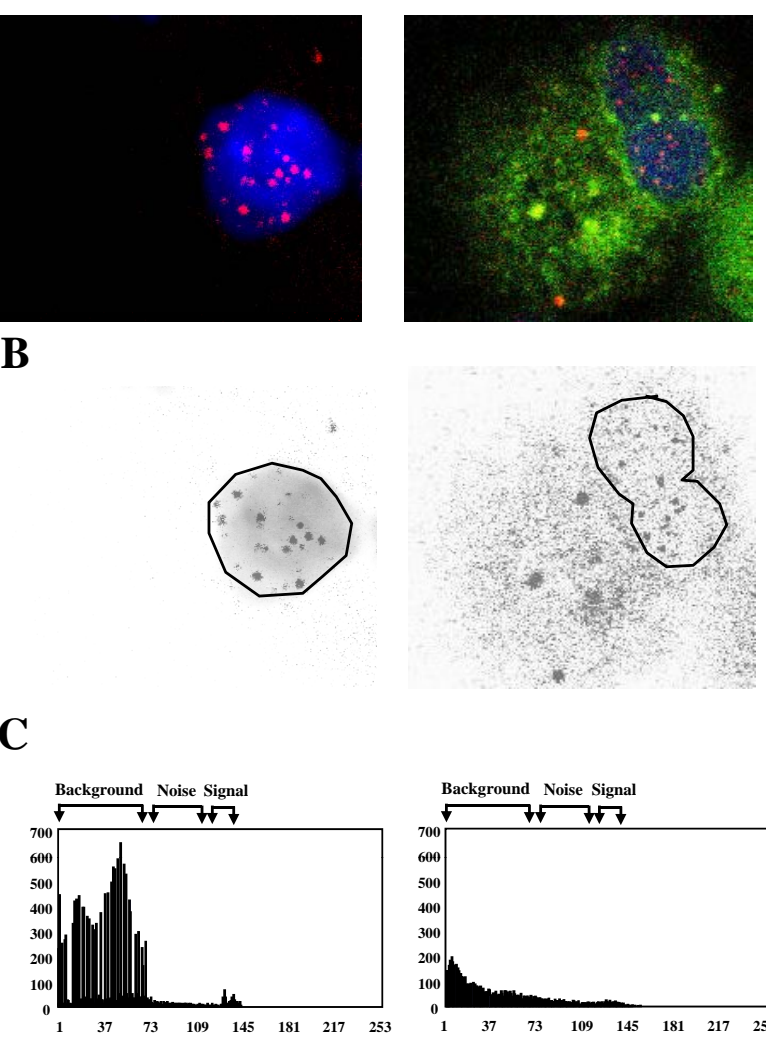

Smoker

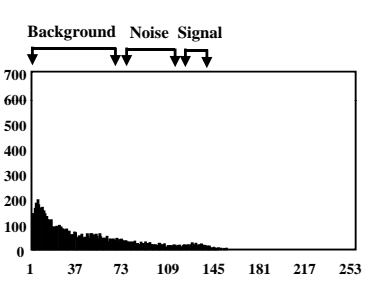

Fig. (2). Image analyses of quantitative fluorescence in situ hybridization (QFISH) performed using NIH image analysis software. Digitized images from alveolar macrophages were obtained using confocal microscopy (A). Black- white images were produced by inverting the image from the red channel (B). The cell nuclei were manually selected by the operator. The telomere fluorescence intensity was analyzed using spot-finding method $(\mathbf{C})$. The background red fluorescence was calculated as the average intensity of all non-spot pixels within each nucleus under consideration and this value was subtracted from each pixel within a spot to yield background corrected red within-spot pixel intensities.

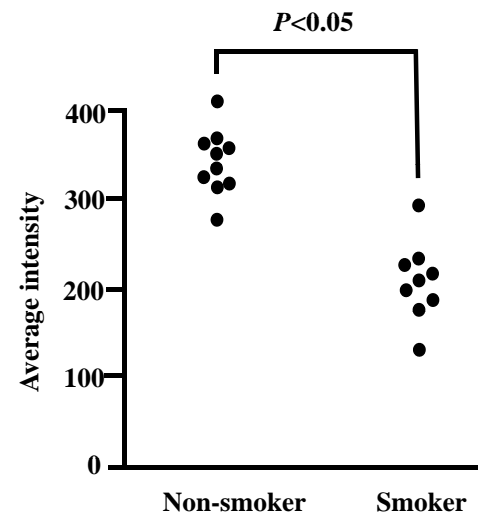

Fig. (3). Telomere length is reduced in alveolar macrophages of young smokers. The intensity of telomere fluorescence from bronchoalveolar lavage macrophages was analyzed using the spotfinding method. Approximately 50 cells were analyzed at each population doubling level (PDL) to yield an average telomere fluorescence of signals. 
length seen in smokers versus non-smokers may not be due simply to signal loss from high background autofluorescence.

\section{A}
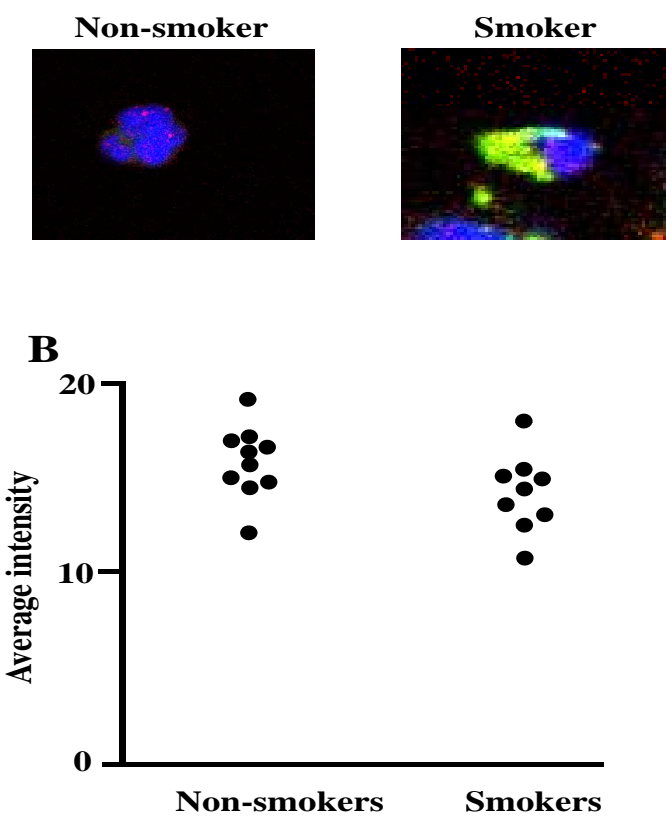

Fig. (4). Centromere length is not affected in alveolar macrophages of young smokers. Confocal microscope images of quantitative fluorescence in situ hybridization (QFISH) using centromere (red) probe counterstained with a fluorescent DNA dye, DAPI, in alveolar macrophages (AMs) from non-smokers and smokers (A). Red indicates centromere signals obtained with rhodamine-labeled probe targeted at the centromeric region of chromosome 8 (CEN8), and blue indicates DAPI-stained chromosomal DNA. The intensity of centromere fluorescence from bronchoalveolar lavage macrophages was analyzed using spot-finding method and shown graphically for each subject (B).

Telomere shortening limits the number of cell divisions of primary human cells and might affect the regenerative capacity of organ systems during aging and chronic disease. Previous studies have shown that increased oxidative stress accelerates telomere shortening [27]. In vitro studies show that telomere shortening is accelerated by oxidative stress through the formation of 8-oxodG at the GGG triplet in the telomere sequence [28]. We hypothesized that smoking induced oxidative stress in the airway, resulting in DNA damage at the telomeric sites. In this study, loss of telomeric repeats in AMs from both younger and older smokers may limit the number of cell divisions possible in precursor cells and reflect an enhanced aging of these terminally differentiated cells possibly leading to abnormal cellular function.

Measurement of telomere length is considered a surrogate maker of cellular senescence [29]. Indeed, estimation of telomere length in human peripheral blood lymphocytes from healthy human donors has shown an inverse correlation between telomere length and age [30, 31]. To determine whether cells are senescent or not, it is necessary to measure not only telomere shortening but also the expression of cyclin-dependent kinase inhibitors such as $\mathrm{p} 21$ or $\mathrm{p} 16$, and senescence-associated $\beta$-galactosidase. We have previously shown that tobacco smoking enhances $\mathrm{p} 21$ and p16 expression in human AMs [4]. Tsuji and colleagues reported that epithelial cells from COPD showed upregulation of $\mathrm{p} 21$ and $\mathrm{p} 16$, telomere length shortness, and inhibition of apoptosis [18]. These results suggest that tobacco-associated diseases, such as COPD, might contribute to senescence of AMs and epithelial cells.

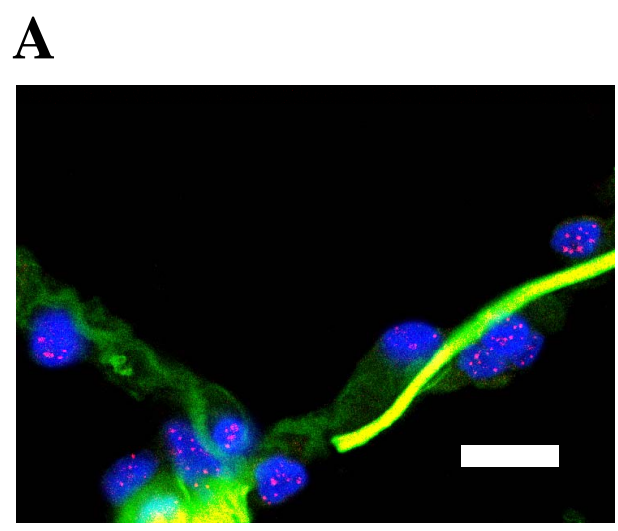

B
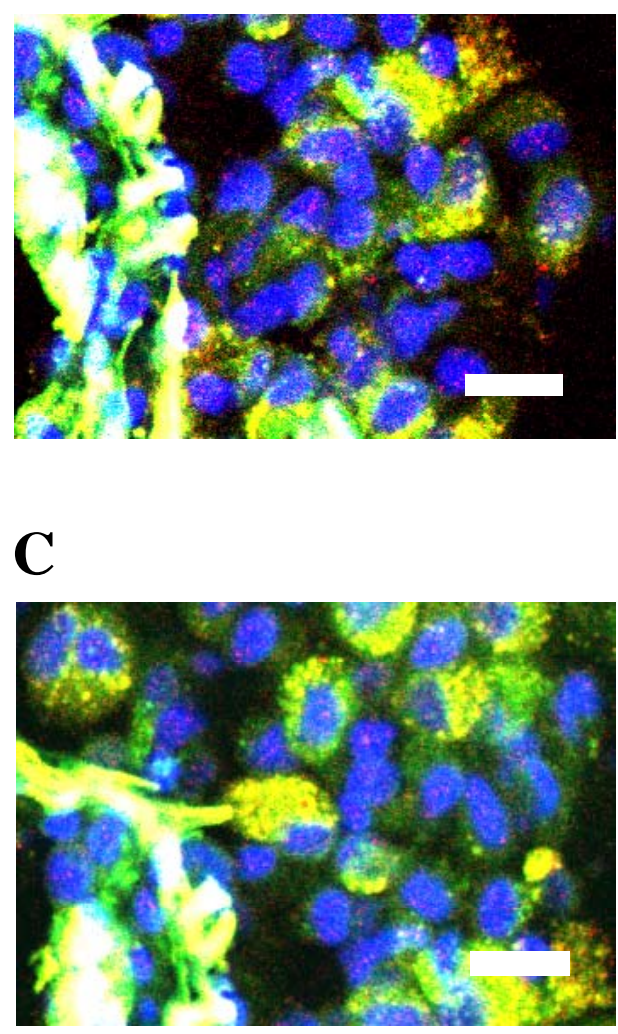

Fig. (5). Representative confocal microscope images of quantitative fluorescence in situ hybridization (QFISH) using telomere (red) probe counterstained with a fluorescent DNA dye, DAPI, in lung tissues from age-matched healthy non-smokers (A), healthy smokers (B) and COPD patients (C). Lung tissues were stained for telomeres and DAPI. Red indicates telomere signals obtained with Cy3-labeled (CCCTAA) $)_{3}$ PNA, and blue indicates DAPI-stained chromosomal DNA. Scale bar $=100 \mu \mathrm{m}$ for each panel. 


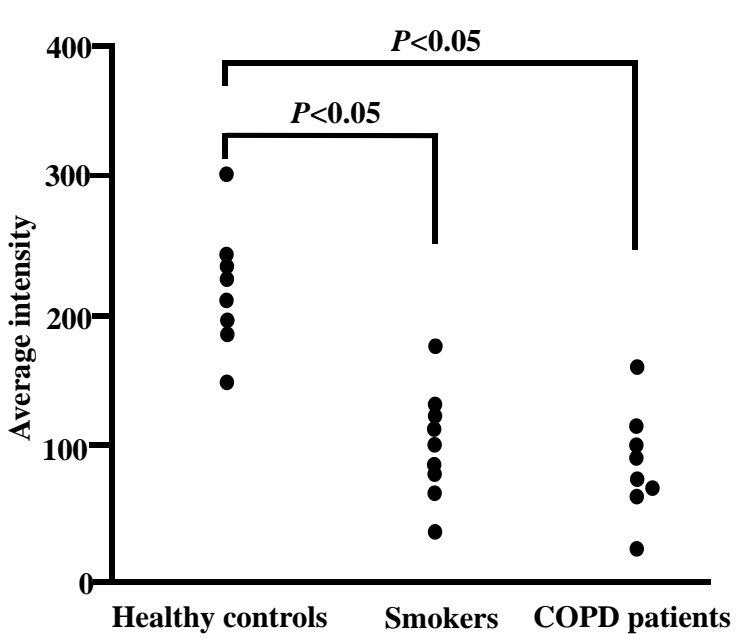

Fig. (6). Telomere length shortening in lung biopsy sections in agematched healthy controls, healthy smokers, and COPD patients using peptide nuclei acid (PNA) telomere probes. Telomere lengths are expressed as image analysis algorithms of a background value subtraction method. Approximately 50 cells were analyzed at each population doubling level (PDL) to yield an average telomere fluorescence.

Morla and colleagues have previously reported that telomere length was reduced in peripheral blood lymphocytes in smoker's with and without COPD [11]. Their evidence suggested that tobacco smoking might indirectly affect AM telomere length. As AMs originate from peripheral blood monocytes, peripheral blood monocytes with shortened telomeres were suggested to be preferentially recruited into airways before differentiated into AMs. Our results suggested that telomere shortening of smoking-exposed AMs might not contribute to susceptibility of smokers to COPD per se as a similar decrease in teleomere length was seen smokers with and without obstruction. However, senescent AMs might contribute to the slow destruction of alveolar walls in smokers. A further study examining this in patients with or without emphysema may clarify this issue.

\section{CONCLUSION}

We provide the evidence that smoking induces shortening of telomere length in AMs from both young and old subjects. Our findings suggest that smoking might induce AMs senescence and COPD might be associated with premature aging. Although the functional role effect of this alteration has still to be delineated, it may be involved in the increased susceptibility of smokers to COPD, communityacquired pneumonia, and lung carcinoma and warrants further investigation.

\section{ABBREVIATIONS}
AMs $=$ Alveolar macrophages
CAP $=$ Community-acquired pneumonia
$\mathrm{CO}=$ Carbon monoxide
COPD $=$ Chronic obstructive pulmonary disease
$\mathrm{FEV}_{1}=$ Forced expiratory volume in one second
FVC $=$ Forced vital capacity

IIPs $=$ Idiopathic interstitial pneumonias

PNA $=$ Peptide nucleic acid

QFISH $=$ Quantitative fluorescence in situ hybridization

$\mathrm{TRF}=$ Terminal restriction fragment

\section{REFERENCES}

[1] Tetley TD. Macrophages and the pathogenesis of COPD. Chest 2002; 121: S156-S9.

[2] Thomas ED, Ramberg RE, Sale GE, et al. Direct evidence for a bone marrow origin of the alveolar macrophages in man. Science 1976; 192: 1016-7.

[3] Marques LJ, Teschler H, Guzman J, et al. Smoker's lung transplanted to a nonsmoker. Am J Respir Crit Care Med 1997; 156: $1700-2$.

[4] Tomita K, Caramori G, Lim S, et al. Increased $\mathrm{p} 21^{\mathrm{CIP} 1 / \mathrm{WAF} 1}$ and $\mathrm{B}$ cell lymphoma leukemia-xL expression and reduced apoptosis in alveolar macrophages from smokers. Am J Respir Crit Care Med 2002; 166: 724-31.

[5] Blackburn EH. Structure and function of telomeres. Nature 1991; 350: 69-73.

[6] Harley CB, Futcher AB, Greider CW. Telomeres shorten during ageing of human fibroblasts. Nature 1990; 345: 458-60.

[7] von Zglinicki T, Burkle A, Kirkwood TB. Stress, DNA damage and ageing - an integrative approach. Exp Gerontol 2001; 36: 1049-62.

[8] Minamino T, Miyauchi H, Yoshida $T$, et al. Endothelial cell senescence in human atherosclerosis: role of telomere in endothelial dysfunction. Circulation 2002; 105: 1541-44.

[9] Samani NJ, Boultby R, Butler R, et al. Telomere shortening in atherosclerosis. Lancet 2001; 358: 472-3.

[10] von Zglinicki T, Serra V, Lorenz M, et al. Short telomeres in patients with vascular dementia: An indicator of low antioxidative capacity and a possible risk factors? Lab Invest 2000; 80: 1739-47.

[11] Morla M, Busquests X, Pons J, et al. Telomere shortening in smokers with and without COPD. Eur Respir J 2006; 27: 525-8.

[12] Valdes AM, Andrew T, Gardner JP, et al. Obesity, cigarette smoking, and telomere length in women. Lancet 2005; 366: 662-4.

[13] Hoxha M, Dioni L, Bonzini M, et al. Association between leukocyte telomere shortening and exposure to traffic pollution: A cross-sectional study on traffic officers and indoor office workers. Environ Health 2009; 8: 41

[14] Tsakiri KD, Cronkhite JT, Kuan PJ, et al. Adult-onset pulmonary fibrosis caused by mutations in telomerase. Proc Natl Acad Sci USA 2007; 104: 7552-7.

[15] Alder JK, Chen JJ, Lancaster L, et al. Short telomeres are a risk factor for idiopathic pulmonary fibrosis. Proc Natl Acad Sci USA 2008; 105: 13051-6.

[16] Savale L, Chaouat A, Bastuji-Garin S, et al. Shortened telomeres in circulating leukocytes of patients with chronic obstructive pulmonary disease. Am J Respir Crit Care Med 2009; 179: 566-71.

[17] MacNee W. Accelerated lung aging: a novel pathogenic mechanism of chronic obstructive pulmonary disease (COPD). Biochem Soc Trans 2009; 37: 819-23.

[18] Tsuji T, Aoshiba K, Nagai A. Alveolar cell senescence in patients with pulmonary emphysema. Am J Respir Crit Care Med 2006; 174: 886-93.

[19] Pauwels RA, Buist AS, Ma P, et al. Global strategy for the diagnosis, management, and prevention of chronic obstructive pulmonary disease: national heart lung, and blood institute and world health organization global initiative for chronic obstructive lung disease (GOLD): executive summary. Am J Respir Crit Care Med 2001; 46: 798-825.

[20] American Thoracic Society. Recommendations for standardized procedures for the online and offline measurements of exhaled lower respiratory nitric oxide and nasal nitric oxide in adults and children. Am J Respir Crit Care Med 1999; 160: 2104-17.

[21] Haslam PL, Baughman RP. Report of ERS Task Force: guidelines for measurement of acellular components and standardization of BAL. Eur Respir J 1999; 14: 245-8.

[22] Ito K, Ito M, Elliott WM, et al. Decreased histone deacetylase activity in chronic obstructive pulmonary disease. N Engl J Med 2005; 352: 1967-76.

[23] Harley CB, Futcher AB, Greider CW. Telomeres shorten during ageing of human fibroblasts. Nature 1990; 345: 458-60. 
[24] Slijepcevic P. Telomere length and telomere-centromere relationships? Mutat Res 1998; 404: 215-20.

[25] Permer S, Brüderlein S, Hasel C, et al. Quantifying telomere lengths of human individual chromosome arms by centromerecalibrated fluorescence in situ hybridization and digital imaging. Am J Pathol 2003; 163: 1751-56.

[26] O'Sullivan JN, Finley JC, Risques RA, et al. Telomere length assessment in tissue sections by quantitative FISH: Image analysis algorithm. Cytometry 2004; 58A: 120-31.

[27] von Zglinicki T. Role of oxidative stress in telomere length regulation and replicative senescence. Ann NY Acad Sci 2000; 908: 99-110.
[28] Kawanishi S, Oikawa S. Mechanism of telomere shortening by oxidative stress. Ann NY Acad Sci 2004; 1019: 278-84.

[29] Sherr CJ, DePinho RA. Cellular senescence: mitotic clock or culture shock? Cell 2000; 102: 407-10.

[30] Hastie ND, Dempster M, Dunlop MG, et al. Telomere reduction in human colorectal carcinoma and with ageing. Nature 1990; 346: 866-68.

[31] Rufer $\mathrm{N}$, Brummendorf $\mathrm{TH}$, Kolvraa $\mathrm{S}$, et al. Telomere fluorescence measurements in granulocytes and $\mathrm{T}$ lymphocyte subsets point to a high turnover of hematopoetic stem cells and memory T cells in early childhood. J Exp Med 1999; 190: 157-67.

(C) Tomita et al.; Licensee Bentham Open.

This is an open access article licensed under the terms of the Creative Commons Attribution Non-Commercial License (http: //creativecommons.org/licenses/by-nc/ 3.0/) which permits unrestricted, non-commercial use, distribution and reproduction in any medium, provided the work is properly cited. 